\title{
THE STONE-ČCH COMPACTIFICATION OF THE RATIONAL WORLD
}

\author{
by M. P. STANNETT $\dagger$
}

(Received 12 September, 1986)

1. Introduction. In his paper [11], Peter Neumann considered in detail the cycle structures of elements of Aut $(\mathbb{Q})$, the group of all homeomorphisms of the "rational world" $\mathbb{Q}$ onto itself, and further analyses of $\operatorname{Aut}(\mathbb{Q})$ and its subgroups have been given by Mekler [9], Bruyns [1], and Truss [13]. My interest in Aut $(\mathbb{Q})$ stems from its utility in proving an at first sight rather startling (to a general topologist) result concerning $\beta \mathbb{Q}$, the so-called Stone-Čech compactification of $\mathbb{Q}$, namely that $\beta \mathbb{Q} \backslash \mathbb{Q}$ is separable, and in fact contains a homogeneous countable dense subspace. (A space $X$ is "homogeneous" provided whenever $x, y \in X$, there is some $g \in \operatorname{Aut}(X)$ with $g(x)=y$.) This is in sharp contrast to the spaces $\beta \mathbb{N} \backslash \mathbb{N}$ and $\beta \mathbb{R} \backslash \mathbb{R}$, which are both inseparable.

From the point of view of this paper, the easiest way to think of $\beta \mathbb{Q}$ is simply as a compact Hausdorff space which just happens to contain a dense copy of $\mathbb{Q}$. There are many other such spaces, of course, including such apparently "nice" spaces as the interval $[0,1]$, but the reason we've picked upon $\beta \mathbb{Q}$ is that every element of $\operatorname{Aut}(\mathbb{Q})$ actually extends to a homeomorphism of the whole of $\beta \mathbb{Q}$ onto itself. This extension property will be easily derived from the following well-known definition of the Stone-Cech compactification of an arbitrary Tychonov space $X$. (A space is "Tychonov" provided it exists as a subspace of a compact Hausdorff space, so that for example $\mathbb{Q}$ is Tychonov.)

EXTENSION PRINCIPLe. Let $X$ be a Tychonov space. Then $\beta X$ is that compact Hausdorff space such that

(a) $\beta X$ contains a dense copy of $X$; and

(b) if $Y$ is any compact Hausdorff space and $f: X \rightarrow Y$ any continuous mapping, then there exists a unique continuous extension $f^{\beta}: \beta X \rightarrow Y$ such that the following diagram commutes.

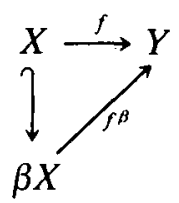

For a proof that $\beta X$ exists, and is essentially unique, as well as an informative review of its structure, consult e.g. Walker's classic text [14].

It follows, of course, that Aut $(\mathbb{Q})$ has a natural action on $\beta \mathbb{Q}$, and it is this action with which we shall be mainly concerned. For example, we shall see that, given any point

$\dagger$ The author would like to acknowledge the support of the SERC during the preparation of this paper.

$$
\text { Glasgow Math. J. } 30 \text { (1988) 181-188. }
$$


$x$ of $\beta \mathbb{Q} \backslash \mathbb{Q}$ the stabiliser of $x$ in Aut(Q) is highly transitive on $\mathbb{Q}$. It will then follow that Aut $(\mathbb{Q})$ has no countable orbit in $\beta \mathbb{Q}$, except $\mathbb{Q}$ itself.

I should like to take this opportunity to express my sincerest thanks to Peter Neumann for his helpful and stimulating correspondence concerning Aut(Q).

2. The action of Aut $(\mathbb{Q})$. We begin by proving our claim that Aut $(\mathbb{Q})$ acts both on $\beta \mathbb{Q}$ and $\beta \mathbb{Q} \backslash \mathbb{Q}$. In fact, we'll see that $\operatorname{Aut}(\mathbb{Q})$ and $\operatorname{Aut}(\beta \mathbb{Q})$ are isomorphic in a very natural way.

LEMMA 1. Every homeomorphism $g \in \operatorname{Aut}(\mathbb{Q})$ has a unique extension $g^{\beta} \in \operatorname{Aut}(\beta \mathbb{Q})$. Moreover, the map $\theta: g \rightarrow g^{\beta}$ is a group isomorphism $\operatorname{Aut}(\mathbb{Q}) \rightarrow \operatorname{Aut}(\beta \mathbb{Q})$.

Proof. Each $g$ in Aut $(\mathbb{Q})$ can be thought of as a continuous map $g: \mathbb{Q} \rightarrow \beta \mathbb{Q}$, so the extension principle implies the existence of a continuous extension $g^{\beta}: \beta \mathbb{Q} \rightarrow \beta \mathbb{Q}$. Our task is to show that $g^{\beta}$ is actually a homeomorphism.

This follows almost immediately, since $g^{-1}$ also has a continuous extension $\left(g^{-1}\right)^{\beta}: \beta Q \rightarrow \beta Q$. Now $g^{\beta} \circ\left(g^{-1}\right)^{\beta},\left(g^{-1}\right)^{\beta} \circ g^{\beta}$ and $i d_{\beta Q}$ are all continuous extensions to $\beta \mathbb{Q}$ of the embedding id: $\mathbb{Q} \rightarrow \beta \mathbb{Q}$, whence they are equal, by the uniqueness clause of the extension principle. Thus $g^{\beta}$ and $\left(g^{-1}\right)^{\beta}$ are both bijections, and are mutual inverses, whence our claim follows.

The uniqueness of extensions also implies that $\theta: g \rightarrow g^{\beta}$ is well-defined, while the equality $g=\left.\theta(g)\right|_{Q}$ shows that $\theta$ is injective. Since $\theta$ is clearly a homomorphism, we need only show that it is also surjective.

To see this, we appeal to a result of Cech [2], that no point of $\beta \mathbb{Q} \backslash \mathbb{Q}$ can have a countable neighbourhood base. Since every point of $\mathbb{Q}$ has such a base (because $\mathbb{Q}$ is a metric space), $\mathbb{Q}$ must be a union of $\operatorname{Aut}(\beta \mathbb{Q})$-orbits in $\beta \mathbb{Q}$. Thus whenever $h$ lies in $\operatorname{Aut}(\beta \mathbb{Q})$, we have $\left.h\right|_{\mathbb{Q}} \in \operatorname{Aut}(\mathbb{Q})$, whence $h=\theta\left(\left.h\right|_{\mathbb{Q}}\right)$, and $\theta$ is surjective.

It follows immediately from this lemma that Aut $(\mathbb{Q})$ acts naturally on $\beta \mathbb{Q}$. To see that $\operatorname{Aut}(\mathbb{Q})$ also acts on $\beta \mathbb{Q} \backslash \mathbb{Q}$ we simply note that $\beta \mathbb{Q} \backslash \mathbb{Q}$ is a union of $\operatorname{Aut}(\beta \mathbb{Q})$-orbits in $\beta \mathbb{Q}$, because $\mathbb{Q}$ is, and so there is a homomorphism

$$
\phi: \operatorname{Aut}(\mathbb{Q}) \rightarrow \operatorname{Aut}(\beta \mathbb{Q} \backslash \mathbb{Q})
$$

given by $\phi(g):=\left.g^{\beta}\right|_{\beta Q \mathbb{Q}}$. In fact, since $\beta \mathbb{Q} \backslash \mathbb{Q}$ is dense in $\beta \mathbb{Q}$-an immediate corollary of our next result-the map $\phi$ is actually an injection.

The action of Aut $(\mathbb{Q})$ on $\beta \mathbb{Q}$ and $\beta \mathbb{Q} \backslash \mathbb{Q}$ cannot be transitive, because Aut $(\mathbb{Q})$ has $c$ elements, while $\beta \mathbb{Q}$ and $\beta \mathbb{Q} \backslash \mathbb{Q}$ both have $2^{c}$ elements $[\mathbf{5}, 9.3]$. However, the action is "very nearly" transitive, in that each orbit of Aut $(\mathbb{Q})$ is dense in its respective space, as we now show.

Note that our proof of Lemma 1 shows that given any Tychonov space $X, \operatorname{Aut}(X)$ has a natural action both on $\beta X$ and $\beta X \backslash X$.

THEOREM 2. Let $X$ be a Tychonov space, and suppose that $H \leqq \operatorname{Aut}(X)$. If there exists a base $\mathbb{B}$ for the topology on $X$ satisfying

(i) $\forall B_{1}, B_{2} \in \mathbb{B}, \exists h \in H$ such that $h\left(B_{1}\right) \subseteq B_{2}$ 
and then

(ii) $\beta X=\bigcup\left\{\bar{B}^{\beta X}: B \in \mathbb{B}\right\}$,

(a) all $H$-orbits in $\beta X \backslash X$ are dense in $\beta X \backslash X$,

(b) all $H$-orbits in $\beta X$ are dense in $\beta X$.

Proof. If $X$ is compact, then $X=\beta X$, so that (a) is trivial, while (b) is an immediate consequence of (i). Suppose then that $X$ is non-compact, and choose $x \in \beta X \backslash X$ and $B_{1} \in \mathbb{B}$ with $x \in \bar{B}_{1}^{\beta X}$. Let $U$ be any non-empty open subset of $\beta X \backslash X$, and choose $V$, an open subset of $X$, with $U \cup V$ open in $\beta X$. Since $\beta X$ is compact Hausdorff, we can choose $W$, a non-empty open set in $\beta X$, with $W \subseteq \bar{W}^{\beta X} \subseteq U \cup V$. Now $X$ is dense in $\beta X$, so that $W \cap X$ is nonempty, and we may choose $B_{2} \subseteq W \cap X\left(B_{2} \in \mathbb{B}\right)$. Let $h \in H$ satisfy $h\left(B_{1}\right) \subseteq B_{2}$.

Put $h^{*}:=\left.\left(h^{\beta}\right)\right|_{\beta X \backslash X}$. Then

$$
h^{*}(x)=h^{\beta}(x) \in h^{\beta}\left(\bar{B}_{1}^{\beta X}\right) \subseteq \bar{B}_{2}^{\beta X} \subseteq \bar{W}^{\beta X} \subseteq U \cup V .
$$

Since $h^{*}(x) \notin V$, we must have $h^{*}(x) \in U$. This proves (a).

To prove part (b), let $W_{1}$ be any non-empty open set in $\beta X$, and put $U=W_{1} \cap$ $(\beta X \backslash X)$ and $V=W_{1} \cap X$. Choose any $x \in \beta X$, and $B_{1} \in \mathbb{B}$ with $x \in \bar{B}_{1}^{\beta X}$. As above, choose $B_{2} \in \mathbb{B}$ satisfying $\bar{B}_{2}^{\beta X} \subseteq U \cup V$, and $h$ satisfying $h\left(B_{1}\right) \subseteq B_{2}$. Once again, we have $h^{\beta}(x) \in \bar{B}_{2}^{\beta X} \subseteq W_{1}$.

COROLLARY 3. There exists a countable subgroup $H$ of Aut $(\mathbb{Q})$ such that

(a) every $H$-orbit in $\beta \mathbb{Q}$ is dense,

(b) every $H$-orbit in $\beta \mathbb{Q} Q$ is dense.

In particular, then, if $q \in \beta \mathbb{Q} \backslash \mathbb{Q}$, there exists a countable dense homogeneous set $\Delta_{q} \subseteq \beta \mathbb{Q} \backslash \mathbb{Q}$, with $q \in \Delta_{q}$.

Proof. We choose $\mathbb{B}$ to be the collection of all proper nonempty clopen subsets of $\mathbb{Q}$, of the form

\section{$\mathbb{Q} \cap(I \cup J)$}

where $I$ and $J$ are intervals (in $\mathbb{R}$ ) with boundary (in $\mathbb{R}$ ) contained in $\sqrt{2} \mathbb{Q} \backslash\{0\}$. Note that $\mathbb{B}$ is countable, so that we can put $\mathbb{B}=\left\{B_{n}: n \in \mathbb{N}\right\}$.

Now $\beta \mathbb{Q}=\bigcup\left\{\bar{B}_{n}^{\beta Q}: n \in \mathbb{N}\right\}$. To see this, let $\left(q_{\lambda}\right)$ be a net in $\mathbb{Q}$ converging to $x$ in $\beta \mathbb{Q}$, and let $U \subseteq \beta \mathbb{Q}$ be a proper open neighbourhood of $x$. According to $[5,16 \mathrm{~F}, 16.11]$, $\beta \mathbb{Q}$ has a base consisting of clopen sets, and so there is a proper clopen neighbourhood $V$ of $x$ such that $x \in V=\bar{V}^{\beta Q} \subseteq U$.

Let $\left(q^{\prime}\right)$ be the net $\left(q_{\lambda}\right) \cap V$; i.e. the part of $\left(q_{\lambda}\right)$ lying in $V$. Then $q^{\prime} \rightarrow x$ and $\left(q^{\prime}\right) \subset V \cap \mathbb{Q}$. The latter is clopen in $\mathbb{Q}$, and nonempty. Moreover it is proper, lest $\beta \mathbb{Q}=\overline{\mathbb{Q}}^{\beta \mathbb{Q}} \subseteq \bar{V}^{\beta \mathbb{Q}} \subseteq U \neq \beta \mathbb{Q}$.

Consequently we can find a superset $B$ of $V \cap \mathbb{Q}$ of the desired form, i.e. with $B \in \mathbb{B}$, and now $x \in \bar{B}^{\beta Q}$.

So $\mathbb{B}$ satisfies condition (ii) of Theorem 2 . We now choose $H$ to satisfy condition (i). 
For each $(i, j) \in \mathbb{N} \times \mathbb{N}$, choose $h_{i, j} \in \operatorname{Aut}(\mathbb{Q})$, satisfying

$$
h_{i, j}\left(B_{i}\right) \subseteq B_{j} .
$$

To see that such $h_{i, j}$ exist, observe that it follows from [11, Sierpinski's Theorem] that any two non-empty clopen subsets of $\mathbb{Q}$ are homeomorphic. Hence there are homemorphisms from $B_{i}$ to $B_{j}$ and from $\mathbb{Q} \backslash B_{i}$ to $\mathbb{Q} \backslash B_{j}$ which can be combined to give a suitable $h_{i, j}$.

We now set $H=\left\langle h_{i, j} \mid i, j \in \mathbb{N}\right\rangle$. Since $H$ is countably generated, it is countable, and by Theorem 2, the $H$-orbits in $\beta \mathbb{Q}$ and $\beta \mathbb{Q} \backslash \mathbb{Q}$ are dense.

In particular, if $q \in \beta \mathbb{Q} \backslash \mathbb{Q}$, set $\Delta_{q}=\{h(q): h \in H\}$. Then $\Delta_{q} \subseteq \beta \mathbb{Q} \backslash \mathbb{Q}$ is countable, dense, homeogeneous, and contains $q$.

An immediate consequence of Corollary 3 is that $\beta \mathbb{Q} \backslash \mathbb{Q}$ is dense in $\beta \mathbb{Q}$, since any $H$-orbit in $\beta \mathbb{Q} \backslash \mathbb{Q}$ is simultaneously an $H$-orbit in $\beta \mathbb{Q}$.

In fact, we could have shown the separability of $\beta \mathbb{Q} \backslash \mathbb{Q}$ quite differently, using the idea of $\pi$-weight. The proof we now give of the separability of $\beta \mathbb{Q} \backslash \mathbb{Q}$ and its density in $\beta \mathbb{Q}$ does not show that the countable dense subset of $\beta \mathbb{Q} \backslash \mathbb{Q}$ can be chosen to be homogeneous, but has advantages in the scope of its application.

3. The iterated remainders of $\mathbb{Q}$. The space $\beta X \backslash X$, where $X$ is a Tychonov space, is called the growth or remainder of $X$ and is usually denoted $X^{*}$. This space is itself Tychonov, and so we can consider $\left(X^{*}\right)^{*},\left(\left(X^{*}\right)^{*}\right)^{*}$, and so on. This notation is rather cumbersome, and so we introduce the following alternative.

Put $X^{(0)}:=X$, and for each $n \in \mathbb{N}$, define $X^{(n+1)}:=\left(X^{(n)}\right)^{*}$. Both Jackson [7] and Hussak [6] have considered those spaces for which $X^{(n)}$ is eventually empty for some $n$. In fact, $\mathbb{Q}^{(n)}$ is never empty for any $n$, and is "so non-empty" that $\mathbb{Q}^{(n+1)}$ is actually dense in $\beta \mathbb{Q}^{(n)}$, for all $n \in \mathbb{N}$. We prove this now. We'll need the following definition.

A space $X$ is said to be "nowhere locally compact" provided every compact subset of $X$ has empty interior. In particular, the space $\mathbb{Q}$ is nowhere locally compact.

LeMma 4. For $n \in \mathbb{N}, \mathbb{Q}^{(n)}$ is nowhere locally compact.

Proof. We proceed by induction on $n$. It's already known that $\mathbb{Q}^{(0)}$ is nowhere locally compact, so we assume that $\mathbb{Q}^{(n)}$ is nowhere locally compact. Suppose $\mathbb{Q}^{(n+1)}$ fails to be nowhere locally compact, so that there is some non-empty open set $U$ in $\mathbb{Q}^{(n+1)}$, and $\bar{U}^{\mathbb{Q}^{(n+1)}}$ is compact. Choose some open $W$ in $\beta \mathbb{Q}^{(n)}$ with $U=W \cap \mathbb{Q}^{(n+1)}$. Now $\mathbb{Q}^{(n)}$ is dense in $\beta \mathbb{Q}^{(n)}$, so that $W \cap \mathbb{Q}^{(n)}$ is nonempty. Moreover, $\bar{U}^{\mathbb{Q}^{(n+1)}}$ is closed in $\beta \mathbb{Q}^{(n)}$, so that $W \backslash \bar{U}^{\mathbb{Q}^{(n+1)}}$ is open in $\beta \mathbb{Q}^{(n)}$, nonempty, and lies entirely in $\mathbb{Q}^{(n)}$. But now, since $\beta \mathbb{Q}^{(n)}$ is compact Hausdorff, we can find some open nonempty $V \subseteq W \backslash \bar{U}^{Q^{(n+1)}}$ with $V \subseteq \bar{V}^{\beta Q^{(n)}} \subseteq$ $W \backslash \bar{U}^{\mathbb{Q}^{(n+1)}} \subseteq \mathbb{Q}^{(n)}$. So $\bar{V}^{\beta \mathbb{Q}^{(n)}}$ is a compact subspace of $\mathbb{Q}^{(n)}$ with nonempty interior. But this contradicts our hypothesis that $\mathbb{Q}^{(n)}$ is nowhere locally compact.

Corollary 5. For each $n \in \mathbb{N}, \mathbb{Q}^{(n+1)}$ is dense in $\beta \mathbb{Q}^{(n)}$.

Proof. If $\mathbb{Q}^{(n+1)}$ isn't dense in $\beta \mathbb{Q}^{(n)}$ for some $n$, we can find a non-empty open set $U$ in $\beta \mathbb{Q}^{(n)}$ lying entirely in $\mathbb{Q}^{(n)}$. But now $U$ contains compact sets with nonempty interior, so that $\mathbb{Q}^{(n)}$ fails to be nowhere locally compact. 
A $\pi$-base for the topology on a space $X$ is a collection $\mathbb{P}$ of nonempty open sets in $X$ such that every nonempty open set in $X$ contains a member of $\mathbb{P}$. The $\pi$-weight of a space $X, \pi(X)$, is the smallest cardinal of any $\pi$-base for $X$.

If $X$ is a Tychonov space, and $Y$ is a dense subspace of $X$ then $\pi(Y)=\pi(X)$. (See e.g. [8].) Any space of countable $\pi$-weight is separable: just pick one point from each member of some countable $\pi$-base; the resulting countable set is dense.

COROllaRy 6. For each $n \in \mathbb{N}, \mathbb{Q}^{(n)}$ is separable.

Proof. We show, by induction on $n$, that $\pi\left(\mathbb{Q}^{(n)}\right)$ is countable for each $n$.

Clearly $\pi(\mathbb{Q})=\aleph_{0}$. Suppose, therefore, that $\pi\left(\mathbb{Q}^{(n)}\right)=\aleph_{0}$. Then $\pi\left(\beta \mathbb{Q}^{(n)}\right)=$ $\pi\left(\mathbb{Q}^{(n)}\right)=\aleph_{0}$, since $\beta \mathbb{Q}^{(n)}$ is Tychonov and $\mathbb{Q}^{(n)}$ is dense in $\beta \mathbb{Q}^{(n)}$. But $\mathbb{Q}^{(n+1)}$ is also dense in $\beta \mathbb{Q}^{(n)}$, by Corollary 5 , whence $\pi\left(\mathbb{Q}^{(n+1)}\right)=\pi\left(\beta \mathbb{Q}^{(n)}\right)=\aleph_{0}$.

4. Aut $(\mathbb{Q})$ orbits in $\beta \mathbb{Q}$. Recently, John Truss ["The group of autohomeomorphisms of $\mathbb{Q}$ : subgroups of small index"-personal correspondence] has succeeded in proving Neumann's conjecture [3] that whenever $H$ is a subgroup of Aut(Q) with $\mid$ Aut(Q) $: H \mid<2^{\aleph_{0}}$, then $H$ necessarily contains the pointwise stabiliser of some finite set in $\mathbb{Q}$. This conjecture also prompted the question whether there exists a countable orbit of Aut(Q) in $\beta \mathbb{Q} \backslash \mathbb{Q}$ such that if $G$ is the stabiliser of a point in this orbit then $G$ does not contain the stabiliser of finitely many points in $\mathbb{Q}$. Truss's result shows that this is not the case, and in fact we can go further; we show that $\beta \mathbb{Q} \backslash \mathbb{Q}$ contains no countable Aut $(\mathbb{Q})$-orbits, so that the question becomes redundant. Before proving this, we shall need the following results of Peter Bruyns [1].

Let $H$ be a subgroup of index $<2^{\aleph_{0}}$ in $\operatorname{Aut}(\mathbb{Q})$. Then

(A) there is a finite subset $Y_{0}$ of $\mathbb{Q}$ that is invariant under $H$, and such that $\mathbb{Q} \backslash Y_{0}$ is a single $H$-orbit,

(B) if $H$ is transitive on $\mathbb{Q}$ (i.e. if $Y_{0}$ is empty) then $H=\operatorname{Aut}(\mathbb{Q})$.

Consequently, as Neumann points out [personal correspondence], if we could find a point of $\beta \mathbb{Q}$ whose Aut $(\mathbb{Q})$-orbit is countable, then its stabiliser in Aut $(\mathbb{Q})$ would have finite orbits in $\mathbb{Q}$.

Let $\tau: \mathbb{Q} \rightarrow \mathbb{Q} \cap(0,1)$ be a homeomorphism. According to the extension principle, $\tau$ has a continuous extension $\tau^{\beta}: \beta \mathbb{Q} \rightarrow[0,1]$, and in fact $\tau^{\beta}(\beta \mathbb{Q} \backslash \mathbb{Q})=[0,1] \backslash(\mathbb{Q} \cap(0,1))$ $[14,6.12]$.

THEOREM 7. Let $A$ be any finite subset of $[0,1] \backslash(\mathbb{Q} \cap(0,1))$. Then the pointwise stabiliser of $\left(\tau^{\beta}\right)^{-1}(A)$ in Aut $(\mathbb{Q})$ is highly transitive on $\mathbb{Q}$.

Proof. Let $A:=\left\{a_{1}, \ldots, a_{n}\right\}$. Putting $G:=\operatorname{Aut}(\mathbb{Q})$, we have to show that $G_{\left(\tau^{\beta}\right)^{-1}(A)}$ is $m$-transitive on $\mathbb{Q}$, for each $m$.

Let $\left(b_{1}, \ldots, b_{m}\right)$ and $\left(c_{1}, \ldots, c_{m}\right)$ be two $m$-tuples of distinct points in $\mathbb{Q}$. Then $\tau^{\beta}\left(b_{i}\right)$ and $\tau^{\beta}\left(c_{i}\right)$ do not lie in $A$, for each $i$, so that we can find irrationals $\alpha_{i}, \beta_{i}, \gamma_{i}, \delta_{i}$ with $b_{i} \in\left(\alpha_{i}, \beta_{i}\right)$ and $c_{i} \in\left(\gamma_{i}, \delta_{i}\right)$, and such that the $\left(\alpha_{i}, \beta_{i}\right)$ are mutually disjoint as are the $\left(\gamma_{i}, \delta_{i}\right)$, and each of the $\left(\alpha_{i}, \beta_{i}\right)$ and $\left(\gamma_{i}, \delta_{i}\right)$ are disjoint from $A$. Putting $U_{i}=\left(\alpha_{i}, \beta_{i}\right) \cap \mathbb{Q}$ 
and $V_{i}=\left(\gamma_{i}, \delta_{i}\right) \cap \mathbb{Q}$, let $\phi \in \operatorname{Aut}(\mathbb{Q})$ be any map which interchanges each $U_{i}$ and $V_{i}$, which satisfies $\phi\left(b_{i}\right)=c_{i}$ for each $i$, and which fixes $\mathbb{Q} \backslash\left(\cup U_{i} \cup \cup V_{i}\right)$ pointwise. Any net in $\beta \mathbb{Q}$ converging to a point of $\left(\tau^{\beta}\right)^{-1}(A)$ is eventually outside all of the clopen sets of $\beta \mathbb{Q}$ whose intersection with $\mathbb{Q}$ are $U_{i}, V_{i}$, whence $\left(\tau^{\beta}\right)^{-1}(A)$ is fixed pointwise by $\phi^{\beta}$, and our claim is proven.

CoRollary 8. There are no countable Aut $(\mathbb{Q})$-orbits in $\beta \mathbb{Q}$ except $\mathbb{Q}$ itself.

Proof. This is immediate from Theorem 7, together with Neumann's interpretation of Bruyns' results, discuissed earlier.

5. Special points and subspaces of $\beta \mathbb{Q}$. We saw in Corollary 5 that $\mathbb{Q}^{(n+1)}$ is dense in $\beta \mathbb{Q}^{(n)}$, for each $n$. It follows from the extension principle that we can find surjections

$$
f_{n}: \beta \mathbb{Q}^{(n+1)} \rightarrow \beta \mathbb{Q}^{(n)}
$$

fixing $\mathbb{Q}^{(n+1)}$ pointwise, and in fact $f_{n}\left(\mathbb{Q}^{(n+2)}\right)=\mathbb{Q}^{(n)}[14,6.12]$. Now the spaces $\mathbb{Q}$ and $\mathbb{Q}^{*}$ are both real compact $[5,8 H]$, non-compact spaces, which must therefore fail to be pseudocompact [5,5H2]. Since the continuous image of a pseudocompact space is again pseudocompact, we see that none of the spaces $\mathbb{Q}^{(n)}$ can be pseudocompact.

Frolik [4] has shown that whenever $X$ is a non-pseudocompact Tychonov space, then $X^{*}$ fails to be homogeneous. Thus, we have shown

Proposition 9. None of the spaces $\mathbb{Q}^{(n)}(n \geqq 1)$ is homogeneous.

Since $\mathbb{Q}^{*}$ isn't homogeneous, it makes sense to consider what "kinds of points" occur in $\beta \mathbb{Q}$ and $\mathbb{Q}^{*}$.

A point $x$ in $\mathbb{Q}^{*}$ is said to be remote provided it lies in the closure of no discrete subspace of $\mathbb{Q}^{*}$ not already containing $x$, and is a weak $P$-point provided it lies in the closure of no countable subset of $\mathbb{Q}^{*}$ not already containing $x$. Since $\mathbb{Q}^{*}$ is separable, by Corollary $3, \mathbb{Q}^{*}$ contains no weak $P$-points. On the other hand Plank [12] has shown, using the Continuum Hypothesis, that $\beta \mathbb{Q}$ contains $2^{c}$ remote points which form a dense subspace of $\mathbb{Q}^{*}$. Since the homeomorphic image of a remote point is again remote, this density is obvious from Corollary 3 , and indeed a homogeneous countable dense subspace of $\mathbb{Q}^{*}$ exists comprising only remote points of $\beta \mathbb{Q}$. Using Martin's Axiom [:= MA], van Mill [10] has shown the existence of a point $x_{0}$ of $\mathbb{Q}^{*}$ which lies in the closure of no countable nowhere dense set in $\beta \mathbb{Q}$ not already containing $x_{0}$.

We can use van Mill's result to construct two interesting subspaces of $\mathbb{Q}^{*}$.

EXAmpLE 10.[MA]. There exists a compact subspace $F$ of $\mathbb{Q}^{*}$ with a point $x_{0} \in \overline{F \backslash\left\{x_{0}\right\}}{ }^{\beta \infty}$ not in the closure of any countable subset of $F \backslash\left\{x_{0}\right\}$.

To see this, enumerate $\mathbb{Q}$ as $\left\{q_{n}: n \in \mathbb{N}\right\}$. Since $\beta \mathbb{Q}$ is Hausdorff, we can find, for each $n \in \mathbb{N}$, a neighbourhood $U_{n}$ of $q_{n}$ with $x_{0} \notin{\overrightarrow{U_{n}}}^{\beta Q}$. Let $V$ be any open neighbourhood of $x_{0}$ in $\beta \mathbb{Q}$, and define

Put $F=\bigcap\left\{\bar{F}_{n}{ }^{\beta Q}: n \in \mathbb{N}\right\}$.

$$
F_{n}:=V \backslash \bigcup_{j=0}^{n} \bar{U}_{j}^{\beta Q}
$$


Since each $\bar{F}_{n}{ }^{\beta Q}$ is a closed neighbourhood of $x_{0}, F$ is a nonempty compact subset of $\beta \mathbb{Q}$. By construction $F$ misses $U_{n}$ for each $n \in \mathbb{N}$, so that $F \subseteq \mathbb{Q}^{*}$.

And since $F$ misses $\mathbb{Q}, F$ contains no non-empty open subset of $\beta \mathbb{Q}$, i.e. $F$ is nowhere dense in $\beta \mathbb{Q}$.

By the choice of $x_{0}, x_{0}$ is not in the closure of any countable subset of $F \backslash\left\{x_{0}\right\}$, since subsets of nowhere dense sets are themselves nowhere dense. exist.

However, $x_{0} \in \overline{F \backslash\left\{x_{0}\right\}^{\beta Q}}$, lest $x_{0}$ be a $G_{\delta}$ in $\mathbb{Q}^{*}$; it is shown in [2] that no such points

EXAmple 11 . There exists a space $X$ which is countable, homogeneous, dense-initself (since dense in $\beta \mathbb{Q} \backslash \mathbb{Q}$ ), of countable $\pi$-weight, $T_{4}$, paracompact, Lindelof and zero-dimensional, but nowhere first-countable.

Moreover, if [MA] is assumed, then every nowhere dense subspace of $X$ is closed, and hence discrete.

Proof. Let $H$ be the group constructed in Corollary 3, and take $X$ to be any $H$-orbit in $\mathbb{Q}^{*}$. Then $X$ is countable and homogeneous. Since $X$ is dense in $\mathbb{Q}^{*}$, we have $\pi(X)=\pi\left(\mathbb{Q}^{*}\right)=\aleph_{0}$, and $X$ is zero-dimensional, since $\mathbb{Q}^{*}$ is; $[5,16 F, 16.11]$. Since $X$ is countable, it's paracompact (because Lindelof and Tychonov $[15,20.8]$ ) and so $T_{4}$ $[15,20.10]$. Finally, since no point of $\mathbb{Q}^{*}$ is the limit of a sequence of distinct points of $\mathbb{Q}^{*}$ $[14,2.2], X$ is nowhere first countable.

Under [MA], we can choose $X$ to contain the point $x_{0}$ of Example 10, whence no point $x \in X$ lies in the closure of any nowhere dense $F \subseteq X$ satisfying $x \notin F$. Thus, every nowhere dense subset of $X$ is closed; since subsets of nowhere dense subsets are again nowhere dense, each nowhere dense subset of $X$ is discrete.

\section{REFERENCES}

1. P. Bruyns; Aspects of the group of homeomorphisms of the rational numbers, Doctoral thesis (Oxford, 1986).

2. E. Čech, On bicompact spaces, Ann. of Math. (2) 38 (1937), 823-844.

3. J. D. Dixon, P. M. Neumann and S. Thomas, Subgroups of small index in infinite symmetric groups, Bull. London Math. Soc., to appear.

4. Z. Frolik, Non-homogeneity of $\beta P \backslash P$, Comment. Math. Univ. Carolin. 8 (1967), 705-709.

5. L. Gillman and M. Jerison, Rings of continuous functions (Van Nostrand, 1960).

6. W. Hussak, Iteratively defined generalisations of locally compact and discrete topological spaces, Doctoral thesis (Sheffield, 1983).

7. P. P. Jackson, Iterated remainders in compactifications, Doctoral thesis (Sheffield, 1980).

8. I. Juhasz, Cardinal functions in topology-ten years later, Math. Centre Tracts 123 (Mathematisch Centrum, Amsterdam, 1980).

9. A. H. Mekler, Groups embeddable in the autohomeomorphisms of $\mathbb{Q}, J$. London Math. Soc. (2) 33 (1986), 49-58.

10. J. van Mill, Weak $P$-points in Čech-Stone compactifications, Trans. Amer. Math. Soc. 273 (1982), 657-678.

11. P. M. Neumann, Automorphisms of the rational world, J. London Math. Soc. (2) 32 (1985), 439-448.

12. D. Plank, On a class of subalgebras of $C(X)$ with applications to $\beta X \backslash X$, Fund. Math. 64 (1969), 41-54. 
13. J. K. Truss, Embeddings of infinite permutation groups, Proceedings of Groups 1985 at $S t$ Andrews (to appear).

14. R. C. Walker, The Stone-Čech compactification, Ergebnisse der Mathematik 83 (Springer, 1974).

15. S. Willard, General Topology (Addison-Wesley, 1970).

Department of Computer SCience

THE UNIVERSITY

SHEFFIELD

S3 7RH 